\title{
REDUCing RUNTIME VALUES IN MiNimum SPANNING TrEe BASED ClUSTERING BY ViSUAL ACCESS TENDENCY
}

\author{
Dr. B. Eswara Reddy ${ }^{1}$ and K. Rajendra Prasad ${ }^{2}$ \\ ${ }^{1}$ Associate Professor, Head of CSE Department, JNTUA College of Engineering \\ Anantapur, Andhra Pradesh, India \\ eswarcsejntu@gmail.com \\ ${ }^{2}$ Research Scholar, JNTUA College of Engineering \\ Anantapur, Andhra Pradesh, India \\ krprgmegmail.com
}

\begin{abstract}
Clustering has been widely used in data analysis. Dissimilarity assesses the distance between objects and this is important in Minimum Spanning Tree (MST) based clustering. An inconsistent edge is identified and removed without knowledge of prior tendency in MST based clustering, which explore the results of clusters in the form of sub-trees. Clustering validity is to be checked at every iterated MST clusters by Dunn's Index. Higher Dunn's Index imposes the exact clustering. The existing system takes more run time when there are several iterations where as the proposed system takes single step with very less run time. Key contribution of the paper is to find prior tendency in MST Based Clustering by Visual Access Tendency (VAT) and to find clustering results in a single step instead of several trails. The proposed method extends the MST based clustering algorithm with VAT procedure, called as VAT-Based-MST-Clustering. Results are tested on synthetic data sets, and real data sets to conclude the clustering results are improved by proposed method with respect to the runtime.
\end{abstract}

\section{KEYWORDS}

Clustering Analysis, Clustering Tendency, Histograms, FFT, VAT

\section{INTRODUCTION}

An unsupervised approach of clustering aims to find related objects for different groups by measuring the features of intra cluster similarity and inter cluster similarity between data objects [3]. Partitional, hierarchical [16], density-based [20], and model based approaches [21] have been developed for clustering analysis [19] and these methods do not perform as expected when the shape of clusters are irregular. But, MST-based clustering algorithm exhibits well even though the shapes of the clusters are complex. Irregular boundaries are handled smoothly in MST-based clustering algorithms [5]. Euclidean distance between every pair of objects introduces the results of dissimilarity matrix. The graph $\mathrm{G}(\mathrm{V}, \mathrm{E})$ is constructed after finding the dissimilarity matrix from given data objects. The MST is a graphical tree analysis and it is derived from graph by standard approaches of either Kruskal's or Prim's algorithm. MST is a fully connected graph of an arbitrary set of data objects. Each dissimilarity value represented as distance between respected data objects; it will act as edge weight between data points in the spanning tree. Cluster separations were effectively formed from MST representation by identifying and removing inconsistent edges. In MST, all longer weighted edges are considered as inconsistent edges. MST-

DOI : 10.5121/ijdkp.2012.2302 
based clustering algorithms [17], [18] create cluster definitions without knowledge of clustering tendency. Because of this reason, the MST algorithm is refined with VAT procedure. VAT computes the reordered dissimilarity matrix for features of data objects. The image of VAT visualizes the tendency value for given dataset [4]. IVAT [1] is extension of VAT approach; it is best suited for finding the clustering tendency on tight clustered datasets. We also make use of different similarity measures for the purpose of finding distance between any two distinguishable objects [6].

VAT and IVAT procedures are made use of the same logic of MST. These procedures reorder the dissimilarity values according to the order of visited vertices during the construction of MST. Clustering assessment is retrieved from VAT image by detecting the number of dark blocks along the diagonal. These dark blocks assess the number of clusters as input of MST based clustering algorithm.

This paper contributes the proposed work on extensive ideas of MST based clustering aiming to extract the tight clusters in order to get two benefits; first is to reduce the time ,and second is to improve the time values since, we use the known tendency value in MST clustering algorithms. The major objective of our proposed research is to make best usage of tendency value in MST based clustering algorithms for improving performance values.

Assessment of tendency is one of the important criteria during clustering analysis. Exact tendency values are inferred from VAT techniques.

Related work of MST-based clustering algorithms is presented in Section 2. Concept of Visual Access Tendency is discussed in Section 3. Proposed work is described in Section 4. Section 5 describes the datasets and its validation. Results analysis is discussed in Section 6, and conclusion and future work is presented in Section 7.

\section{RELATED WORK}

MST based clustering is graph theoretical promising approach for unlabelled data sets. MST is constructed using classical algorithms, namely, either Prim's or Kruskal's. The following sections discussed the construction of MST and their subtree clustering results from dissimilarity features.

\subsection{Deriving MST from unlabeled data}

A spanning tree is to add single edge at a time from the data of graph $G$ with given set of ' $n$ ' number of vertices and ' $\mathrm{e}$ ' number of edges. The edge weight value is obtained from the calculation of Euclidean distance between respective pair of data objects from the unlabelled data. The traditional algorithms either Prim's or Kruskal's are constructed spanning tree from the graph G. They [5] discussed another generic spanning tree algorithm, named REVERSE-DELETE, this algorithm is processed the edges in opposite direction of Prim's or Kruskal's algorithm. REVERSE-DELETE algorithm is to construct the minimum spanning tree from fully connected graph by deleting edges in sorted descending order. The observed cost of these traditional algorithms is $\mathrm{O}(\mathrm{m} \operatorname{logn})$ [7],[8].

\subsection{Overview for MST-Based Clustering}

Removing of an inconsistent edge in MST gives the results of two separated subtrees, which is considered as resulting of two-cluster group. Applying same procedure recursively for each subtree, and ' $\mathrm{K}$ ' clusters are retrieved from the MST. Standard approach of MST-based clustering is reported in Zahn's work [9]. According to Zahn's work, first is to check whether edge weights are larger than sum of average weight of edges and standard deviation of edges, which edges are 
inconsistent edges. Zahn's used the following equation in MST clustering for the purpose of removing inconsistent edges.

$$
\mathrm{W}>\mathrm{W}_{\mathrm{avg}}+\mathrm{W}_{\sigma}
$$

Where $\mathrm{W}$ is inconsistent edge weight, $\mathrm{W}_{\text {avg }}$ be the average weight of edges, and $\mathrm{W}_{\sigma}$ be the standard deviation of edge weights.

$\mathrm{Xu}[10]$ derives MST for representing the gene expression data; Using his approach, we understand that MST-clustering strategy doesn't assume always regular geometric shape. Due to this reason, $\mathrm{Xu}[10]$ describes three objective functions. First objective says simply removing of (K-1)-longer edges, which results total weight of ' $\mathrm{K}$ ' subtrees is minimized. Second objective function states that sum of total distance between pair of center position (center(Ti), Ti is $i^{\text {th }}$ subcluster) and data points(d) is minimized.

$$
\sum_{i=1}^{C} \sum_{d} \operatorname{dis}(d, \text { center }(T i))
$$

Third objective function presents the optimal solution of clustering problem by detecting the best representative of the data set; this function minimize the distance between selected representative and data points in the cluster.

Another approach of MST-based clustering algorithm is proposed by Laszlo and Mukherjee[11], generally this is referred as LM algorithm. They carried out constraint on the clustering size instead of number of clusters. Removing of edges is applied only in case of reaching minimum clustering size.

Vathy-Fogarssy[12] experiments MST-clustering with three basic criterion functions. The objective of this work is to reduce external interference on MST clustering results; First criteria states that delete edge if the edge satisfies the value of attraction threshold.

The Attraction threshold is as follows

$$
\mathrm{T}_{\text {atth }}=0.5^{*} \sqrt{\frac{N(N-1)}{\sum_{i=1}^{N} \sum_{j=i+1}^{N} 1 / d(x i, x j)}}
$$

$\mathrm{N}$ is number of input objects, and the distance between any two objects $\mathrm{d}(\mathrm{x} 1, \mathrm{x} 2)$ is calculated by Euclidean formula. Second criteria detect the hidden separations by recognizing the inconsistent edge, which uses the following condition for detecting inconsistent edge.

$$
\mathrm{d}(\mathrm{xi}, \mathrm{xj})>\mathrm{T}_{\text {atth }}
$$

Third criteria propose largest fuzzy hyper volume is used for the purpose of evaluating good clustering validity. Fuzzy hyper volume is given by the equation

$$
\begin{aligned}
& \left.\sum_{i=1}^{c} V_{i} ; \mathrm{V}_{\mathrm{i}}=\sqrt{\operatorname{det}\left(F U Z_{i}\right.}\right) \\
& \mathrm{FUZ}_{\mathrm{i}}=\frac{\sum_{i=1}^{N}\left(\mu_{i j}\right)^{m}\left(x_{i}-v_{j}\right)\left(x_{i}-v_{j}\right)^{T}}{\sum_{i=1}^{N}\left(\mu_{i j}\right)^{m}}
\end{aligned}
$$

Grygorash [13] proposed Hierarchical Euclidean distance based MST clustering algorithm (HEMST) and the Maximum Standard Deviation Reduction Clustering Algorithm (MSDR). MSDR does not require any input value for termination. MSDR removes edge only when all 
International Journal of Data Mining \& Knowledge Management Process (IJDKP) Vol.2, No.3, May 2012

clusters standard deviation is maximized. This is recursive process and it is continued until the obtained standard reduction is within threshold, but the problem of MSDR is requiring high cost for large datasets. HEMST algorithm generates the clustering results from MST until reaching the required number of clusters. User is giving the input as number of clusters without idea of clustering tendency, which is used as constraint of HEMST algorithm during runtime. Three important limitations are identified in present system. These are clustering tendency is unknown, it doesn't generate the exact number of clusters without knowledge of tendency, and it requires external interference for specifying termination condition.

Therefore, the purpose of assessing tendency, we propose the specific visualization methods in MST based clustering algorithms for detecting exact tendency value. This method is discussed in following sections.

\section{VisuAl ACCESS TENDENCY (VAT) AND IMPROVED VAT (IVAT) METHODS}

VAT is best when the dissimilarity features are available for unlabeled data set. The effective values of dissimilarity results the clarity of tendency in their visual representation. The tendency of data determines itself the cluster numbering from their visual representation by the detecting the number of square shaped dark blocks along the diagonal in the regulating image of VAT algorithm [4]. The following algorithm describes the procedure of VAT.

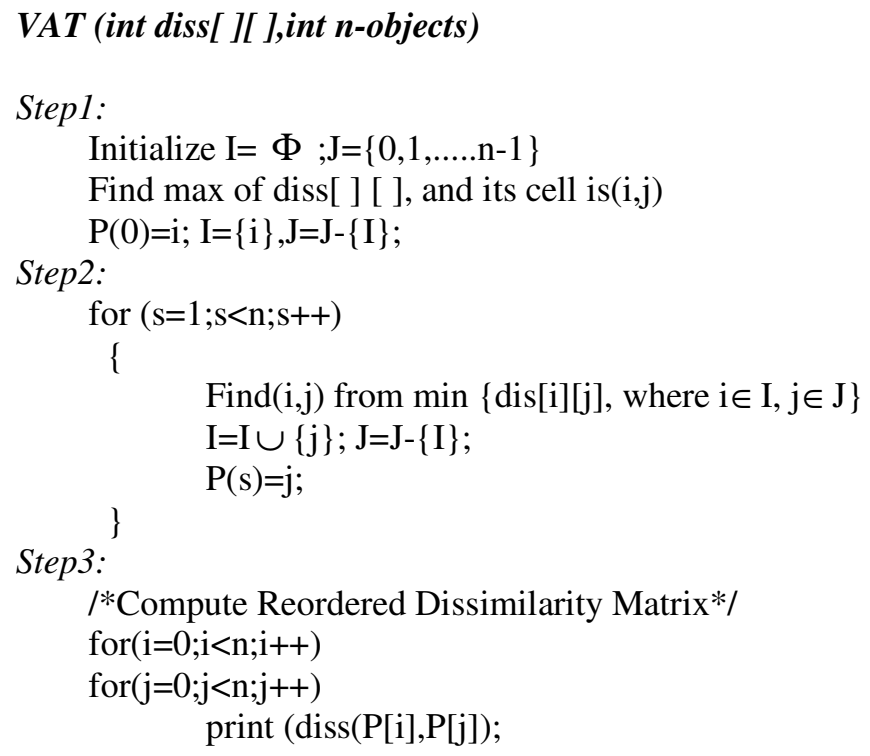

The VAT algorithm visualizes the VAT image of reordered dissimilarity data(diss) from Step3. The results are observed from the following sample dissimilarity data (D).

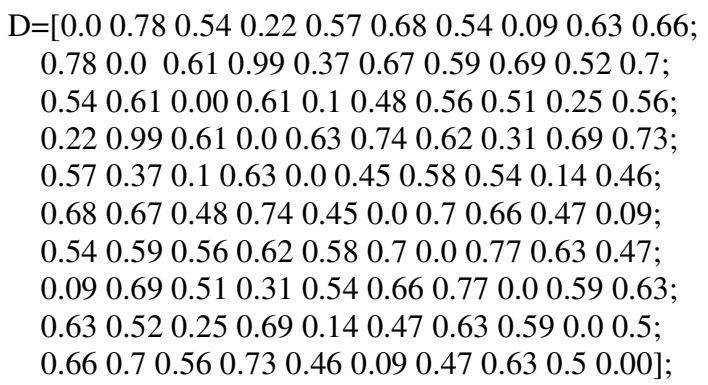




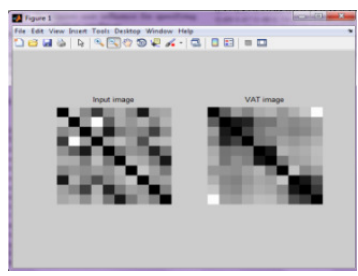

Fig.1. VAT Image for Dissimilarity Matrix (D)

The VAT image results are described in Fig.1. Dissimilarity matrix ' $\mathrm{D}$ ' is derived from unlabeled data set. Fig. 1 illustrates the clustering tendency value (i.e. number of clusters is 5) with the help of number of square shaped dark blocks along the diagonal. This tendency value is used during the process of MST based clustering.

VAT has some limitations before finding the clustering tendency. It performs well when the clustered data is not in complex. The authors of [2] formulate another procedure called as IVAT, which uses graph theoretic distance transform to improve the accuracy of visual clustering tendency. Visual results of VAT and IVAT are presented in the Fig.3 and Fig.4 for the 5-clusterd synthetic data in Fig.2.

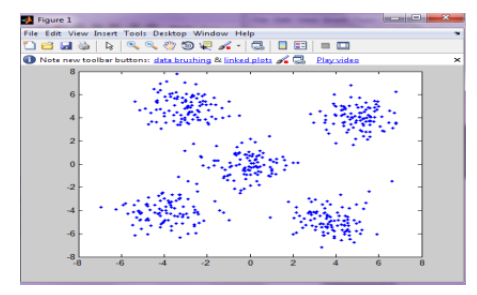

Fig. 2 Five-Clustered Data

5-clustered synthetic data is created in MATLAB editor. VAT and IVAT algorithms are applied on 5-clusterd data sets. MST based clustering algorithm is improvised with IVAT for extracting of correct tendency. Since clarity of visual results are obtained using IVAT version for tough cases data set.

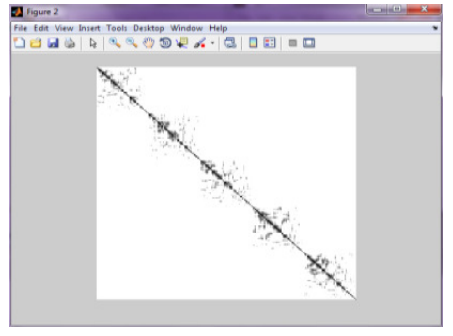

Fig. 3 VAT Image for 5-Clustered Data 


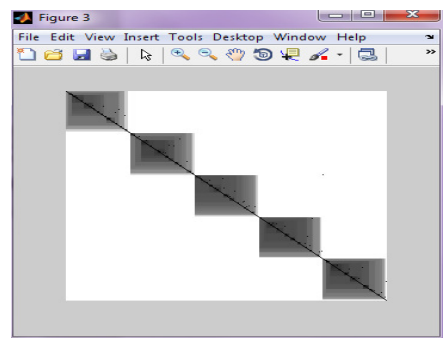

Fig.4 Improved VAT Image for Five-Clustered Data

\section{PROPOSED WORK}

The proposed VAT-Based-MST- Clustering deals with two steps. First step deals the method to explore the value of cluster count value from unlabelled data. Second step deals with the standard procedure of MST clustering where is applied on datasets on the known tendency value is obtained from step1. These steps are highlighted in the proposed method.

We obtain clustering results in our proposed method in a single step instead of taking several trails. Since, prior tendency is extracted from VAT; the following proposition tells that optimal clusters are obtained in proposed VAT-Based-MST-Clustering.

\section{Proposition:}

\section{Higher Dunn's Index imposes optimal MST-clustering results}

\section{Proof:}

Dunn's Index is a good contrast measure of VAT image [14], and it shows well cluster separations (CS)

Dunn's Index $=\frac{N}{M}$

$\mathrm{N}=\min _{1 \leq \mathrm{q} \leq \mathrm{c}} \min _{1 \leq \mathrm{r} \leq \mathrm{c}} \operatorname{dist}\left(\mathrm{C}_{\mathrm{q}}, \mathrm{C}_{\mathrm{r}}\right)$

$\mathrm{M}=\max _{1 \leq \mathrm{p} \leq \mathrm{c}} \operatorname{diam}\left(\mathrm{C}_{\mathrm{p}}\right)$

VAT explores exact partitioning. Dunn's Index is high if the partitioning is correct. MST clustering results are derived from VAT.

Therefore, it proves the proposition.

\section{VAT-Based-MST-Clustering (Input: unlabelled dataset)}

Step1:

a) Find Re-ordered dissimilarity image(I) using either VAT or IVAT

b) Apply Image threshold on I

c) Find histograms by applying consecutive operations of 2D Fast Fourier Transformations, Inverse of Fast Fourier Transformations, and Correlation.

d) Extract the number of square shaped dark blocks as cluster count $\mathrm{c}$. 
Step2:

a) Find Graph 'G' using dissimilarity matrix. An object is act as a node in the graph. Dissimilarity value between pair of objects act as weighted value between the nodes.

b) Construct the minimum spanning tree from Graph ' $G$ ' using any one of the classical algorithm.

c) Detect and remove an inconsistent edges (whose weight value is greater than sum of average and standard deviation of weight values), which results as two sub-trees, treated as two different clusters

d) Step2(c) is repeated until we reach the cluster count 'c' value

\section{DATA SETS AND ITS VALIDATION}

The synthetic data (Fig. 2) is carried out in scattered plot for testing purpose. This scattered data is generated in MATLAB environment by running some data distribution functions. From Fig.2, we have seen data as five clustered groups and the corresponding MST clustering results are obtained during the execution phases of proposed work in our validation. The corresponding MST clustered results are described in the following diagram Fig.5 and Fig.6.

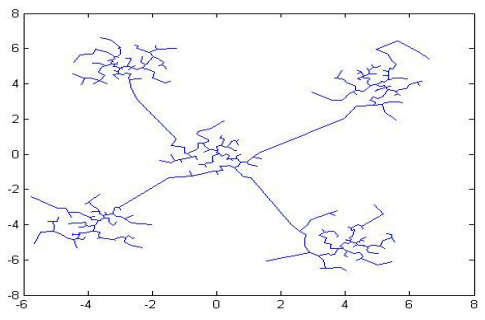

Fig. 5 Minimum Spanning Tree for Five-Clustered Data

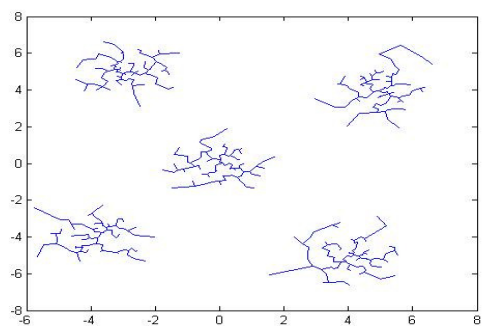

Fig. 6 MST Clusters

\section{RESULT ANALYSIS}

The proposed study has presents the experimental results based on various synthetic data sets (S1, S-2, S-3), which are shown in Fig. 7. 


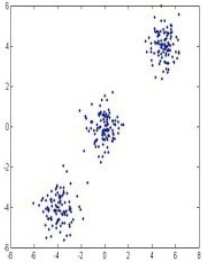

(a) S-1

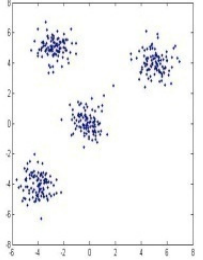

(b) S-2

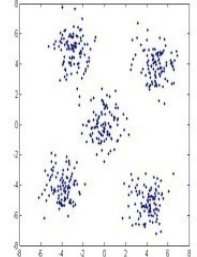

(c) $\mathrm{S}-3$

Fig. 7: Synthetic Datasets

In evaluation of result analysis, the existing system doesn't have the prior value of clustering tendency. External interference is required for clustering tendency. Because of this cause, the obtained results of existing system may or may not have good Dunn's Index. The higher value of Dunn's Index indicates the good number of valid clusters for given data. Dunn's Index is a metric for evaluating of correct partitioning [14]. MST clustering algorithm is experimented several times until getting the good Dunn's Index. So, the problem of existing system is runtime. Therefore, the proposed work first solves the problem of tendency by extracting of obtained square shaped dark blocks, secondly it retrieves MST based clustering results based on tendency.

This procedure output the VAT image for input dataset. After that we apply 2D FFT, IFFT, and correlation on getting VAT image. These steps are described by the authors of [15], and then we obtained histogram in Fig 8. Finally, the clustering number is extracted; it is referred as clustering tendency.

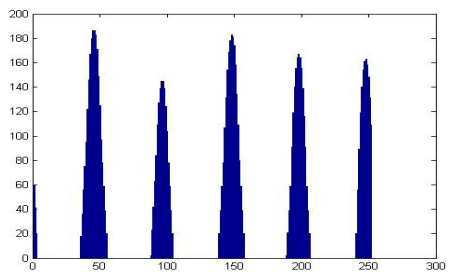

Fig. 8: Histogram for Five Clustered Data

Basic MST clustering procedure uses this tendency value and form sub-tree shaped clusters. Each sub tree cluster has collection of similar objects. These are described in Fig. 6

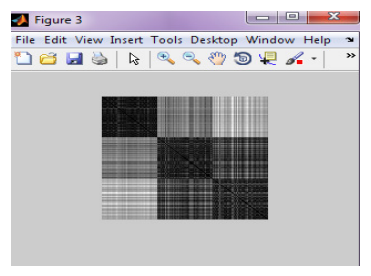

Fig.9a Iris-VAT Image

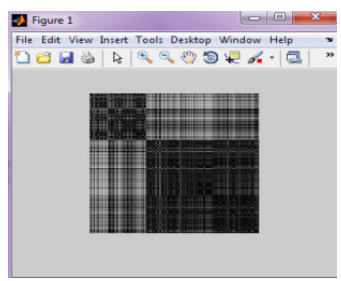

Fig.9b Wine-VAT Image 

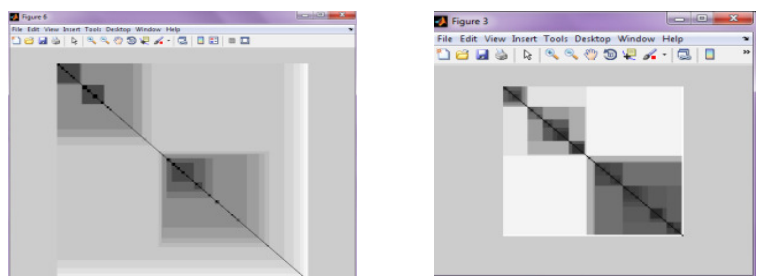

Fig.9c Vote-VAT Image Fig.9d Glass-VAT Image

The running is reduced in new method when compare of existing system. Table 1A and Table 1B show the clustering results and its total runtime for synthetic and real time data sets respectively (the value of Dunn's Index for each cluster). Table 2A and Table 2B shows reduced runtime values for execution of proposed work on both synthetic and real time datasets. In new method, the clustering tendency is derived in single step instead of several trails. Hence, running time is reduced in VAT-Based MST Clustering.

\section{TABLE $1 \mathrm{~A}$}

Dunn's Index and Runtime Results for Synthetic datasets (MST Based Clustering-Clustering Tendency is Unknown)

\begin{tabular}{|c|c|c|c|c|c|c|}
\hline \multirow{2}{*}{$\begin{array}{l}\text { Data } \\
\text { sets }\end{array}$} & \multicolumn{5}{|c|}{$\begin{array}{l}\text { Dunn's Index for Number of } \\
\text { clusters }(\mathrm{C})\end{array}$} & \multirow{2}{*}{$\begin{array}{c}\text { Run } \\
\text { time } \\
\text { in } \\
\text { Sec }\end{array}$} \\
\hline & $\mathrm{C}=2$ & $\mathrm{C}=\mathbf{3}$ & $\mathrm{C}=4$ & $C=5$ & $\mathrm{C}=6$ & \\
\hline S-1 & 0.37 & $\underline{0.55}$ & 0.38 & 0.21 & 0.20 & 5.57 \\
\hline S-2 & 0.26 & 0.26 & $\underline{\mathbf{0 . 5 0}}$ & 0.30 & 0.23 & 8.4 \\
\hline S-3 & 0.23 & 0.26 & 0.21 & $\underline{0.32}$ & 0.25 & 13.4 \\
\hline
\end{tabular}

\section{TABLE 1B}

Dunn's Index and Runtime Results for Real Time Datasets (MST Based Clustering-Clustering Tendency is Unknown)

\begin{tabular}{|c|c|c|c|c|c|c|}
\hline \multirow{2}{*}{ Datasets } & \multicolumn{5}{|c|}{$\begin{array}{l}\text { Dunn's Index for Number of } \\
\text { clusters }(\mathrm{C})\end{array}$} & \multirow{2}{*}{$\begin{array}{c}\text { Run } \\
\text { time } \\
\text { in } \\
\text { Sec }\end{array}$} \\
\hline & $\mathrm{C}=2$ & $\mathrm{C}=\mathbf{3}$ & $C=4$ & $\mathrm{C}=5$ & $\mathrm{C}=6$ & \\
\hline Iris & $\underline{0.33}$ & 0.16 & 0.15 & 0.16 & 0.15 & 1.51 \\
\hline Wine & $\underline{0.10}$ & 0.06 & 0.05 & 0.05 & 0.08 & 1.21 \\
\hline Vote & $\underline{0.39}$ & 0.37 & 0.35 & 0.35 & 0.35 & 2.19 \\
\hline Glass & 0.03 & $\underline{0.058}$ & 0.051 & 0.046 & 0.045 & 1.24 \\
\hline
\end{tabular}


International Journal of Data Mining \& Knowledge Management Process (IJDKP) Vol.2, No.3, May 2012

\section{TABLE $2 \mathrm{~A}$}

Dunn's Index and Runtime Results for Synthetic Datasets (Proposed Approach- Clustering Tendency is Known)

\begin{tabular}{|c|c|c|}
\hline $\begin{array}{c}\text { Synthetic } \\
\text { Datasets }\end{array}$ & $\begin{array}{c}\text { Dunn's Index (Clustering } \\
\text { Tendency 'C' is extracted } \\
\text { from proposed approach) }\end{array}$ & $\begin{array}{c}\text { Runtime } \\
\text { in } \\
\text { Seconds }\end{array}$ \\
\hline S-1 & $0.55(\mathrm{C}=3)$ & 1.36 \\
\hline S-2 & $0.50(\mathrm{C}=4)$ & 2.27 \\
\hline S-3 & $0.32(\mathrm{C}=5)$ & 4.18 \\
\hline
\end{tabular}

TABLE 2B

Dunn's Index and Runtime Results for Real Time Datasets (Proposed Approach- Clustering Tendency is Known)

\begin{tabular}{|c|c|c|}
\hline $\begin{array}{c}\text { Synthetic } \\
\text { Datasets }\end{array}$ & $\begin{array}{c}\text { Dunn's Index (Clustering } \\
\text { Tendency 'C' is extracted } \\
\text { from proposed approach) }\end{array}$ & $\begin{array}{c}\text { Runtime } \\
\text { in } \\
\text { Seconds }\end{array}$ \\
\hline Iris & $0.33(\mathrm{C}=2)$ & 0.71 \\
\hline Wine & $0.10(\mathrm{C}=2)$ & 0.72 \\
\hline Vote & $0.39(\mathrm{C}=2)$ & 1.10 \\
\hline Glass & $0.058(\mathrm{C}=2)$ & 0.61 \\
\hline
\end{tabular}

Fig.10 illustrates the running time comparison results between existing and proposed system for several datasets.
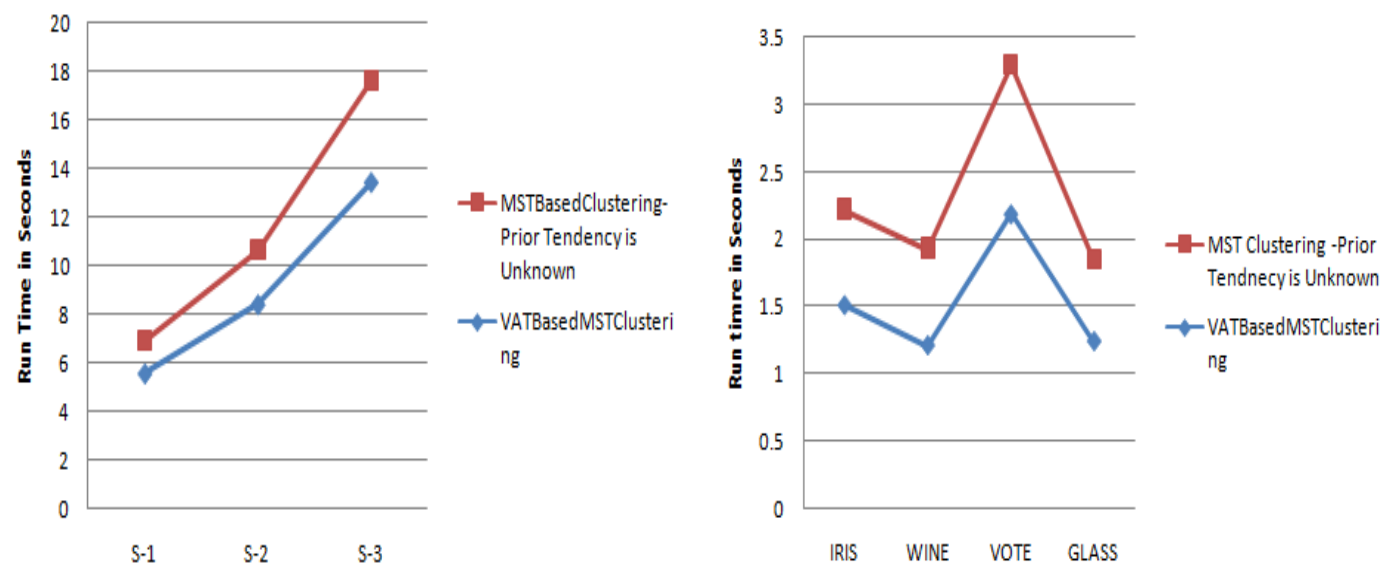

Fig. 10 Runtime Comparison for S-1, S-2, and S-3 


\section{CONCLUSION}

Experimental results are tested on various synthetic datasets. Runtime and Dunns Index values are evaluated and compared in both existing and proposed systems. According to the results analysis, we studied that proposed system requires less time than existing work and also conclude that proposed work produces high quality of clustering results after observing of Dunn's Index value. Dunn's Index measures inter and intra distance values between pair of objects. Higher value of Dunn's Index concludes the good clustering results.

This proposed study majorly includes the ideas of finding tendency and clusters on synthetic data. Spectral approach gives high quality clustering results on complex datasets such as high dimensional data sets. Spectral approach requires high computational cost, but sampling methods reduces this cost. The future scope of the work is to obtain best indexed clustering results by techniques of sampling method and spectral approach in our proposed method.

\section{REFERENCES}

[1] L. Wang, T.Nguyen, J.Bezdek, C. Leckie , and K.Rammohanarao, “iVAT and aVAT: Enhanced visual analysis for clustering tendency assessment" in Proc PAKDD,India, Jun 2010

[2] Timothy C. Havens, James C. Bezdek, " An efficient formulation of the improved visual assessment of cluster tendency" IEEE Trans on Knowledge and Data Engineering,Nov,2011

[3] A. Jain and R.Dubes, “Algorithms for clustering data”, Prentice-Hall,1988

[4] J.Bezdek and R.Hathaway, "VAT: A tool for visual assessment (cluster) tendency", in Proc. IJCNN, Honolulu, Hi,2002, pp.2225-30

[5] Wang,D.Mitchell, “ A Divide-and Conquer Approach for Minimum Spanning Tree-Based Clustering”, Vol21,No 7, July 2009, pp:945-958

[6] M.Kendall and J.D Gibbons, "Rank correlation methods",Newyork,oxford university press, 1990

[7] R.Prim, "Shortest Connection Networks and Some Generalization", Bell systems technical Journal, vol. 36,pp. 1389-1401,1957

[8] J. Kruskal, "On the Shortest Spanning Subtree and the Travelling salesman Problem:, Proc.Am.Math.Soc.,pp 48-50,1956

[9] C.T. Zahn. "Graph theoretical methods for detecting and describing gestalt clusters", IEEE Trans. Computers,vol. 20,no.1, pp. 68-86, Jan. 1971

[10] Ying Xu, Victor Olman, Dong Xu, "Minimum spanning trees for gene expression data clustering", Genome Informatics 12:pg 24-33,2001

[11] M.Laszlo, and S.Mukerjee, "Minimum spanning tree partitioning algorithm for microaggregation", IEEE Trans. Knowledge and Data Engineering, vol 17, no 7, pp 902-911, July 2005

[12] A. Vathy-Fogarassy , A.Kiss, and J.Abnoyi,"Hybrid Minimal Spanning tree based clustering and mixture of Gaussians based clustering algorithm", Foundations of Information and Knowledge systems, pp 313-330, Springer, 2006.

[13] O.Grygorash, Yan Xhpu, Zach Jorgenson, “ Minimum spanning tree based clustering algorithms”

[14] T.C. Havens, J.C.Bezdek, J.M.Keller,M. Popescu, “ Dunn’s Cluster Validity Index as Contrast Measure of VAT Images" Int Conf IEEE 2008

[15] I.J.Sledge, J.M Huband, J.C. Bezdek, “(Automatic) Cluster Count Extraction from Unlabeled Data Sets”, Fifth International Conf. On Fuzzy Systems and Knowledge Discovery, IEEE computer society, pg3-13 
International Journal of Data Mining \& Knowledge Management Process (IJDKP) Vol.2, No.3, May 2012

[16] J.Han and M.Kamber (2002) : Data Mining: concepts and techniques, Elsever

[17] J.L Bentley and J.H Friedman, "Fast algorithms for constructing minimal spanning trees in coordinate spaces”, IEEE Trans computers vol 27 no 2, pp 97-105

[18] R. R.Shamir; D.Tsur,” Cluster graph modification problems. In LNCSI ,pp379-390,springer

[19] ArunK.Pujari:DataMining Techniques”, Universities Press,2001

[20] M.Ester; P. Kriegel; J. Sander; X.xu,” A density based algorithm for discovering clusters in large databases with noise" ,Int Conference on knowledge discovery and data mining 1996 ,pp 226-231

[21] W. Wang; J. Yang; R. Muntz," STING: A statistical information grid approach to spatial data mining", Int Conf on very large data bases, pp 186-195

\section{Authors Biography}

Dr. B. Eswara Reddy Graduated in B.Tech.(CSE) from Sri Krishna Devaraya University in 1995. He received Masters Degree in M.Tech.(Software Engineering), from Jawaharlal Nehru Technological University, Hyderabad, in 1999. He received Ph.D in Computer Science \& Engineering from Jawaharlal Nehru Technological University, Hyderabad, in 2008. He served as Assistant Professor from 1996 to 2006. $\mathrm{He}$ is working as Associate Professor in CSE Dept., since 2006 and currently acting as Head of CSE Dept at Jawaharlal Nehru Technological University Anantapur, Anantapur. He has more than 30 Publications in various International Journals and Conferences. He is one of the author's of the text book titled Programming with Java published by Pearson/Sanguine Publishers. His research interests include Pattern Recognition \& Image Analysis, Data Warehousing \& Mining and Software Engineering. He is a life member of ISTE, IE, ISCA and member of CSI and IEEE

Mr. K. Rajendra Prasad Graduated in B.Tech(CSE) from Jawaharlal Nehru Technological University, Hyderabad in 1999. He received Masters Degree in M.Tech(CSE) from Visvesvaraya Technological University, Belgaum in 2004. Presently, he is a research scholar at JNTUA, Anantapur. He is a life member of CSI. His research interests are data mining \&data warehousing, and databases.

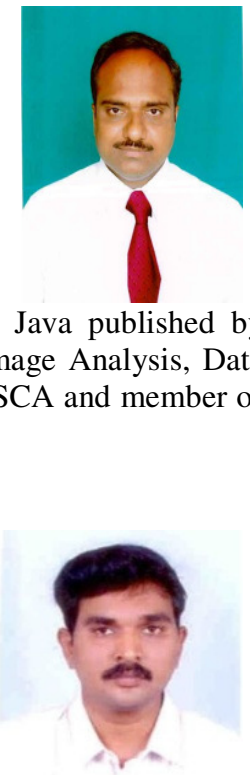

